\title{
Social Determinants of Health Approach to Facilitate Fulfillment of the U.S. Latinx Children's Right to Personal Security: The Strong Communities Initiative as a Case Study
}

\author{
Moore de Peralta A* \\ Department of Languages, Clemson University, USA
}

*Corresponding author: Moore de Peralta Arelis, Assistant Professor of Community Health, Department of Languages, Clemson University; 717 Strode Tower Clemson, SC 29634, USA, Tel: 864-656-3393; Fax: 864-656-0258; Email: ared@clemson.edu

\section{Mini Review}

Volume 5 Issue 1

Received Date: January 15, 2021

Published Date: February 10, 2021

DOI: $10.23880 /$ eij-16000181

\section{Abstract}

Environmental injustice, structural racism, and their effects on poverty affect disproportionately communities of color in the United States (U.S.); contributing to health inequities, including child maltreatment and neglect. Cultural diversity and pluralism makes necessary the study of families' cultural and socioeconomic values to characterize the socio-ecological contexts and social determinants of health (SDOH) to which children from underrepresented communities are exposed to in the U.S. This descriptive paper presents a SDOH approach, by building on the existing literature, to identify socioeconomic, cultural, and contextual conditions that influence Latinx children's fulfillment of their right to personal security. Using the Strong Communities for Children initiative as a case study, a review of practices that, if culturally, linguistically and contextually adapted, could be applicable for promoting Latinx children' safety are discussed. The Strong Communities is a child maltreatment and neglect prevention initiative that promotes the safety and well-being of U.S children by addressing the multilevel conditions or determinants that place children at risk for abuse or neglect. Strong communities is a multilevel, assets-based and socio-ecological approach to preventing child maltreatment and neglect, that incorporates community engagement and capacity building strategies. This approach could facilitate development of culturally and linguistically relevant interventions, by incorporating Latinx cultural values, to promote meaningful engagement of and trust among Latinx immigrant families and communities. The conclusion include a call for capacity building programs and policies that pursue Latinx families' child maltreatment and neglect prevention to use culturally and linguistically relevant, multidisciplinary (E.g. social epidemiology and community-engaged research and interventions), holistic, multilevel, and community-engaged approaches to strengthen children's family and community environment.

Keywords: Children's Right to Personal Security; Child Maltreatment and Neglect; Latinx; Cultural Values; Community Engagement; Social Determinants of Health; Social Epidemiology

Abbreviations: SDOH: Social Determinants of Health; UNDP: United Nations Development Program; TANF: Temporary Assistance for Needy Families; CPS: Child Protective Services; DHHS: Department of Health \& Human
Services; CI: Confidence Interval; CWS: Child Welfare System; ABCD: Assets-Based Community Development; CBPR: Community-Based Participatory Research. 


\section{Epidemiology International Journal}

\section{Introduction}

Environmental injustice, structural racism, and their effects on poverty affect disproportionately communities of color in the United States (U.S) [1]. These socioeconomic and racial social inequities contribute to health inequities [2]. El Sayed A [1] posited that observed racial social inequities, and their implications on poverty, have facilitated the devastation produced by Covid-19 among underrepresented groups in the U.S. Reducing these racial social inequities requires public health approaches focused in the social determinants of health; such as those developed by social epidemiology and community-engaged researchers and practitioners [3]. This descriptive paper presents a social determinants of health (SDOH)-approach and builds on the existing literature, to identify socio-economic, cultural, and contextual conditions that influence the fulfillment of the right to personal security (i.e., physical safety, psychological security, and access to resources necessary for survival and development) for USLatinx children in the U.S. In addition, how the fulfillment of this right could be facilitated by culturally adapting a child maltreatment and neglect prevention initiative (i.e., Strong Communities) [4] that promote the safety and well-being of U.S children by addressing the multilevel conditions or determinants that place children at risk for abuse or neglect [5].

Latinx are the larger minority population group in the U.S [6]. Hence, advancing understanding on culturally relevant adaptation of interventions that promotes the fulfillment of children's right to personal security, including child maltreatment and neglect reduction efforts, may influence the health and wellbeing of children from underrepresented communities in the U.S population. This paper includes a review of practices that, if culturally, linguistically and contextually adapted, could be applicable to contribute with achieving the fulfillment of Latinx children's right to personal security, including their physical safety, their sense of psychological security, and their access to resources sufficient to enable their survival and development [7].

Over the last two decades, the Latinx population across the country experienced a boom [8], growing to about $18 \%$ of the U.S population and making it the largest ethnic or racial minority [6]. The Latinx population growth is of public health relevance, as Latinx are at greater risk than non-Latinx Whites for certain diseases and conditions [9]. These disparities include diabetes [10], cardiovascular disease risks [11], and child maltreatment disparities [12]. In addition, Latinx communities continue to experience ongoing challenges such as anti-immigrant sentiment, immigration raids, racism and discrimination. These challenges result in increased Latinx's mistrust in outside institutions, which may include government entities, hospitals, and universities
[13]. This historical mistrust could be of concern when pursuing community engagement related processes in this population, including those efforts to promote families sense of safety.

Human insecurity is an ancient phenomenon. Threats of famine, war, drought, flood, wild animals, plague, and enslavement appear in ancient writings across the world [14]. The United Nations Development Program's [UNDP] Human Development Report [15] defined human security as,

- "Safety from chronic threats such as hunger, disease and repression"; and

- "Protection from sudden and hurtful disruptions in the patterns of daily life whether in jobs, in homes or in communities."

One of the four UNDP report's essential characteristics of human security stated that human security is easier to ensure through early prevention than later intervention. The relevance of early interventions was reflected in findings of a literature review conducted by Alkire S [14] that showed that human security has been portrayed in the literature as an effect of human interaction with the social determinants of health (SDOH). This is that human security is based on safeguarding, or protecting, human beings against economic, political, health and natural hazards that result, primarily, of the unequal distribution of power, income, goods, and services, globally and nationally, and its consequences on people's lives [16].

The following section presents findings of a review of the literature to portray the importance of culturally and linguistically adapting interventions to promote safety and to prevent child maltreatment and neglect in Latinx communities, by addressing the SDOH at various levels of children's ecological contexts. For this end, the Strong Communities initiative will be used as a case study. The following sections include, a revision of the role of the SDOH and child development's ecological contexts in the fulfillment of children's right to personal security; the situation of child maltreatment and neglect in a racial and ethnically diverse United States' context; the case of the Strong Communities for Children initiative as a multilevel approach to promote children's safety; and finally, some considerations on how to culturally and linguistically adapt interventions to promote children' safety by preventing child maltreatment and neglect.

\section{Fulfillment of Children's Right To Personal Security: The Role of the Social Determinants of Health (SDOH)}

Alkire S [14] argued that human security processes should be consistent with ongoing human development 


\section{Epidemiology International Journal}

efforts by supporting participation, freedom, institutional appropriateness, and diversity. As such, human security is not sufficient for human fulfillment. Gasper D [17] also argued that human security should be seen as the intersection of a concern with reasoned freedoms, a focus on basic needs, and a concern for stability in key human development dimensions. For instance, there is evidence that adequacy of social and material support is critical to children's safety [18]. Findings of a study conducted by Yang MY [19] showed that caregivers receiving Temporary Assistance for Needy Families (TANF), or in general, experiencing material hardship are more likely to become involved in child protective services (CPS) investigations. In general, investigated neglect reports are responsive to particular types of hardship such as housing and food. These types of hardship are addressed in the World Bank's World Development Report 2000/1 on Poverty. This report identified three pillars of poverty reduction efforts: facilitating empowerment, enhancing security, and promoting opportunities. In this report, the World Bank equates security enhancement to addressing the $\mathrm{SDOH}$ when they said that enhancing security means "reducing vulnerability to economic shocks, natural disasters, ill health, disability, and personal violence".

An effort to fulfill the children's right to personal security is collective in nature, as it is multidimensional. It requires a socio-ecological perspective to understand the different social determinants, which in conjunction, influence individual or community development. As such, human security is related with human development because both concepts relate with wellbeing enhancement and are people-centered, multi-sectoral and multidimensional processes. Alkire S [14] argued that human development refers to expanding people's choices or capabilities not only in terms of income, but also in areas such as health, education, technology, the environment, and employment. All these SDOH are intertwined with children's safety.

Other elements of the human security discourse should include a normative focus on individuals' lives and an insistence on basic rights for all, which implies a collective and inclusive impact. At the community level, some preconditions are beneficial to ensure that children' safety efforts are successful. One of these preconditions is social cohesion. Findings of a study on child maltreatment and neglect [20] showed that social cohesion mediated the association between neighborhood-level poverty and abuse rates. The findings suggested that efforts to increase neighborhood social cohesion might be effective in reducing rates of child abuse. These findings are particularly relevant to low-income communities because community members' reach beyond nearby settings may be constrained because of the cost of transportation and relatively limited connections through work and other processes. Therefore, higher levels of social cohesion in a low-income setting might mediate the known negative impact of economic hardships on children' safety. The neighborhoods in which we live may have profound influences on our health, well-being, and ability to function in many realms, including our ability to parent. Neighborhoods that are characterized by layers of disadvantage related to poverty, unemployment, crime, and population turnover could affect the residents in a myriad of ways that are harmful, including increasing risk of child maltreatment [21].

Melton GB [7] posited that the concern for children's safety is intertwined with protection of personal security of historically disadvantaged groups. For instance Jack KM, et al. [12] found that higher levels of socioeconomic disadvantage (poverty, unemployment, and low education), having older children, respondents being male, and respondents being older parents are associated with higher levels of child neglect, while being White is associated with lower levels. The following section covers a discussion on how racial and socio-economic disparities in child maltreatment reflect a disproportionate rate among U.S. minority groups.

\section{Child Maltreatment and Neglect in a Diverse United States Context}

Public health programs aimed at reducing disparities among minority groups are a national priority [22]. Child maltreatment is a significant public health problem. The U.S Administration for Children \& Families [23] informed that of the 3.5 million children who were the subject of an investigation or alternative response in 2017, an estimated 674,000 children were determined to be victims of maltreatment. In total, 74.9 percent of victims were neglected, 18.3 percent were physically abused and 8.6 percent were sexually abused. Given the scale and scope of child maltreatment as a public health issue in the U.S., researchers argued about the importance of identifying factors to prevent child maltreatment and neglect, as well as its potential long-term negative consequences. Particularly, among underrepresented groups which are more affected by prevalent racial social inequities.

Cultural diversity and pluralism have significant implications for service delivery and policymaking [24]. Given the growing multicultural demographic throughout the U.S responsiveness to the particular needs of diverse communities is no longer an option for policy-makers, researchers, and service providers to reduce disparities in the access and utilization of services [25], including child welfare. Particularly, considering documented disparities in the rates at which minority children are exposed to the child 


\section{Epidemiology International Journal}

welfare system in the U.S [12]. Data from 2018, reported by the U.S Department of Health \& Human Services (DHHS), showed that American Indian or Alaska Native children have the highest rate of victimization at 15.2 per 1,000 children in the population of the same race or ethnicity. African American children have the second highest rate at 14.0 per 1,000 children, followed by White $(8.2$ per 1,000$)$ and Latinx (8.1 per 1,000) children.

The U.S. population has become increasingly diverse since the 1990, when the U.S. Advisory Board on Child Abuse and Neglect warned on the fact that the child protection system's focus on reporting and investigation distracted authorities from developing and implementing effective efforts to prevent initial or further harm [5]. The United States' population diversity is reflected particularly with respect to the rapidly growing number of multicultural, multi-lingual low-income children and families. Findings of a study on child maltreatment as a function of poverty and race-ethnicity [26] showed strong positive associations between official child maltreatment and environmental poverty in all race-ethnic groups in the U.S, with Latinx showing lower rates than its Blacks and Whites counterparts do. For instance, at $25 \%$ poverty level, total maltreatment report rates were $6.91 \%$ [95\% confidence interval (CI): $6.43 \%-7.40 \%$ ] for Whites, $6.30 \%$ (5.50\%-7.11\%) for Blacks and $3.32 \%(2.88 \%-3.76 \%)$ for Latinx. Thus, this study's findings showed support to the presence of a "Hispanic paradox" in official maltreatment. Latinx are the higher and fastest growth minority in the U.S, hence, it is important to understand the cultural and other SDOH that contribute with the observed rates of child maltreatment in this population.

\section{Role of Poverty and Culture in Observed Child Maltreatment and Victimization Disparities in the U.S.}

According to Jack KM, et al. [21], racial disparity in child maltreatment remains a significant problem in the U.S., with Black and Latinx children represented in the United States child welfare system (CWS) at rates that are different from their representation in the general population. Although by 2018, Latinx children's rate of victimization was 8.1 per 1,000 children in the population of the same race or ethnicity, which is lower than other race-ethnic groups including Whites (8.2 per 1,000 ) the existing rate of victimization has been related to racial disproportionalities in the poverty population [27]. For all race/ethnic groups, there is continuing evidence that children placed in foster care are predominantly from impoverished families. Pelton LH [27] argument reflects the role of the SDOH in children's safety, and the relationship of the latter with human development. Although the latest poverty rates among Latinx are at a historic low, Latinx continue to be overrepresented among the population in poverty. They made up 18.3 percent of the total population in 2017 , but accounted for 27.2 percent of the population in poverty [28].

The relationship between poverty and child maltreatment disparities is significant. For instance, Jack KM, et al. [21] found that poverty disparities were associated with rates of maltreatment disparities, and densely populated metropolitan counties and sparsely populated counties tended to have the greatest levels of maltreatment disparity for both black and Latinx children. Other study results [19] suggested that in order to prevent child maltreatment, it may be necessary to address a family's unmet material needs through economic support interventions. In addition, Tyrone C, et al. [29] explored risk factors for substantiation maltreatment in three ethnic groups. The authors found that for Latinx, TANF reception lowered substantiation's likelihood, while caregivers' drug use raised its likelihood.

Parents' place of birth is a relevant factor in explaining Latinx children's risk of maltreatment and neglect. Findings of a study by Motoyamaa MJ, et al. [30] showed a protective effect for children's maltreatment and neglect when one of the two of their parents are born outside the U.S, and that this protective effect could be related with cultural behavioral patterns. The authors examined factors associated with substantiation among Latinx children and found that cases involving mixed nativity parents had lower odds of substantiation and that these lower odds might relate to the strengths observed among mixed nativity families, including a lower prevalence of material hardship and active alcohol and drug abuse. The authors called for a culturally relevant child welfare practice with diverse Latinx families.

Findings of previous research [29,30] reflected the importance of reducing poverty disparities, as well as of promoting culturally informed practices to reduce the likelihood of child maltreatment and neglect among Latinx immigrant communities. Particularly, knowing the concern with the loss of social capital among immigrant families who relocate to the U.S [7], as well as the acculturation processes experienced by second and third generation immigrants. Offering culturally and linguistically relevant services and programs is needed to account for Latinx immigrants' loss of social capital and the impact of acculturation processes on children's health and well-being. The next section includes a description, as a case study, of a child maltreatment and neglect prevention initiative (i.e., Strong Communities) [4] that promote the safety and well-being of U.S.-children by addressing the multilevel conditions or determinants that place children at risk for abuse or neglect [5]. 


\section{Epidemiology International Journal}

The Case of the Strong Communities for Children Initiative: A social Determinants of Health Approach to Children's Safety

Strong Communities for Children (From here into "Strong Communities"), funded by the Duke Endowment in 2002, is a multi-year comprehensive community-based initiative to prevent child maltreatment and improve children's safety (for a detailed description of the initiative see McDonell JR, et al. [4]). Strong Communities addresses the multiple individual, family, community and societal level conditions or SDOH (socio-ecological contexts) that place children at risk for abuse or neglect [5]. Strong Communities principles to guide strategic development include

a) Integrating support into settings where children and families are naturally to be found;

b) Strengthening community norms for protecting children;

c) Broadly mobilizing community residents and community leaders to become involved;

d) Strengthening organizational capacity in primary community institution;

e) Helping children by assisting their parents; and

f) Providing support to families and children universally and in non-stigmatizing ways.

Some of the strategies or interventions used in the Strong Communities to contribute with reducing children's risk of abuse or neglect include parents' involvement in their communities, formal and informal social support and interventions to increase social cohesion and mutual support among neighbors. The ultimate goal of Strong Communities is to keep kids safe (i.e., prevent child abuse and neglect) by strengthening participating communities so that "every child and every parent knows that if they have reason to celebrate, worry, or grieve, someone will notice, and someone will care" [18]. Strong Communities was the first large-scale effort to implement strategies proposed in the 1993 report of the U.S. Advisory Board on Child Abuse and Neglect, and it is one of the largest community-wide initiatives ever undertaken for the purpose of improving children's safety in the U.S.

Previous research findings showed that Strong Communities, as a community mobilization strategy, could shift norms of parents' care for their children and neighbors' support for one another, so that young children are safer at home and in the community [4]. Moreover, residents of communities in the Strong Communities service area experienced increased support and help giving, saw their neighborhoods as more cohesive and their neighbors as more willing to pull together, believed their neighbors improved their parenting practices, and improved their own parenting beliefs and parenting practices. In addition, child injuries suggesting child abuse and neglect decreased, and founded cases of child maltreatment declined for children under age
10, the target age for the intervention [4].

Strong Communities research [31] also showed that low-resource communities experienced greater levels of mobilization, as measured by community and institutional engagement, and a greater number of positive outcomes related to changes in the quality of life for families and community norms relative to child and family wellbeing. Considering previous findings showing that Latinx communities are overrepresented among low-income communities [32], if Strong Communities is culturally, linguistically adapted, could likely be a relevant strategy to mobilize, and engaged Latinx communities to prevent child abuse and neglect and to promote children's right to personal security, by addressing the $\mathrm{SDOH}$. The next section includes a review of selected theoretical considerations and approaches that could orient development of culturally and linguistically relevant interventions to prevent child maltreatment and neglect among Latinx immigrant families.

\section{Culturally, linguistically Adapting Interventions to Promote Children' Safety: Child Maltreatment and Neglect Prevention}

Hayden [33] argued that behavior is influenced significantly by culture. Cultural values are "what people hold in high regard, and include normative beliefs regarding all aspects of life including nature, truth, honesty, beauty, education, integrity, friendship, and family" [33). The Latinx culture is rich and diverse. Zea MC, et al. [34] argued that it is not possible to talk about a Hispanic-American perspective or a single Latinx culture. The authors added that diversity among Latinx is based on historical, racial, geographical, linguistic, acculturational and socioeconomic differences. As early as 1995, Baumrind D [35] argued that culture largely determines the inferred basis of parenthood, so that parenting is defined according to cultural understanding and is grounded on the experiences of that culture within its unique environment. Therefore, cultural beliefs and attitudes play a major role in one's health-seeking behavior, health care utilization [36], and parenting practices [37].

There are cross-cultural differences in both parental values and parenting practices [37]. Although all races are represented among Latinx, there are important socioeconomic differences that can be a predictor of attitudes and behaviors as strong as or stronger than cultural heritage [34]. Studying of cultural [37] and socioeconomic [34] values may allow us to identify aspects of parenting that are unique to Latinx and which complement traditional frameworks of parenting. One such framework is the attachment theory [38], which is based on the fundamental idea that human behavior is organized by innate behavioral systems, such as attachment, exploration, caregiving, and sexual mating. Only 


\section{Epidemiology International Journal}

in the context of effective parental care and support could a child develop a stable sense of attachment security, which was viewed as the foundation of optimal child development [39]. Independently of the framework used to orient crosscultural research on parenting, there is a call to gravitate away from research oriented upon assumptions about normative childrearing practices and beliefs that are based on middle-class, European American families [40].

For the purpose of this paper, we will focus on two relevant Latinx cultural values that may influence parenting style and practices and neighborliness, which are acculturation and familism. Acculturation has been widely used as a research variable to measure the effects of changes in beliefs, behavior, and values in health, as well as to study how these effects may change as individuals begin to integrate some of the values of the mainstream culture [41]. Familism is "a cultural value that involves individuals' strong identification with and attachment to their nuclear and extended families, and strong feelings of loyalty, reciprocity, and solidarity among members of the same family" [42]. Familism is related to acculturation among Latinx. Even highly acculturated Latinx held more familistic attitudes than White non-Latinx [43]. Earlier research findings [44] showed that individuals who reported higher levels of familism were more likely to engage in healthy behaviors and less likely to practice risky ones. Efforts to implement interventions to promote positive parenting practices among Latinx should take in consideration the acculturation level of participants, as well as to incorporate familism as an overarching value to guide design and implementation of such interventions.

Efforts to eliminate health disparities in Latinx communities could be moved forward by building capacity in these communities, with culturally appropriate and community engaged research methods/approaches [13,45]. Multidisciplinary approaches to community development have been developed based on the impact of community and societal factors on health outcomes [46].

For instance, community development efforts to prevent child maltreatment and neglect that incorporate some type of capability approach or capacity building (i.e., which similarly re-orients the objective of economic activity from an instrumental end [economic growth] to a people-centered end would be relevant to Latinx immigrant communities. This capability approach would facilitate combining human safety with human development approaches, as stated in the UNDP Human Development Reports, which used the language of capabilities and functioning and describe the capability approach as "expanding people's choices". As stated by Melton GB [7], child protection efforts, including those to protect children's personal security, that include community action are especially significant when they result not only in protection of the right to personal security per se, but also in vindication of other dimensions important to their survival, development, and well-being.

Capacity building programs and policies that pursue Latinx families' child maltreatment and neglect prevention should be holistic, multilevel approaches that strengthen the children's family environment to enhance children' safety and to improve their own, their parents', and their neighbors' quality of life. Such approaches should also incorporate Latinx families and communities' cultural values and assets. Strong Communities is an example of an approach that uses assets in the community to expand and strengthen networks of supportive relationships and, in particular, to increase support to parents.

Strong Communities is an assets-based community development (ABCD) initiative, and as such, it is a valuable approach to engage Latinx communities in efforts to prevent child maltreatment and neglect by tapping into existing strengths and resources in these communities (E.g., familism, mutual support, neighborliness, etc.). Communitybased participatory research (CBPR) [47] and community engagement are other capacity building approaches, which involve working with and through groups of people to change their environment and behavior in order to improve their health. Ahmed SM, et al. [48] defined community engagement in research as "a process of inclusive participation that supports mutual respect of values, strategies, and actions for authentic partnership of people affiliated with or selfidentified by geographic proximity, special interest, or similar situations to address issues affecting the well-being of the community of interest". Findings of a study [12] showed that higher levels of mother's community involvement were associated with lower levels of child psychological aggression. Similarly, that more positive perception of community social control was associated with lower levels of child physical assault. The authors suggested that building positive and supportive communities is a protective factor in child maltreatment. In addition, that engaging parents in their communities can improve the environment in which children and families develop, and decrease the likelihood that maltreatment will occur.

The Strong Communities initiative incorporates community engagement, which is a strategy that can amplify the voices of those at risk of losing their right to personal safety, as well as to incorporate Latinx community members' input into the design and implementation of interventions to prevent child maltreatment and neglect. The experience of community members observing and, better, directly participating in community activities to promote child and family well-being reinforces the belief, individually and collectively, that action on behalf of families will be effective 


\section{Epidemiology International Journal}

and promotes trustworthy relationships between community members and outside institutions. Such experiences stimulate the perception that the community is a welcoming and supportive place and the expectation that the experience for families in the community will be positive [18]. CBPR and community engagement can be used to mediate mistrust among underrepresented populations [49]; and, as such, of particular relevance for Latinx immigrant families that has often reported increased mistrust in outside institutions as a result of anti-immigrant laws, racism, and discrimination expressed by members of other population groups.

\section{Recommendations to Incorporate Latinx Communities Culture and Context into Research and Programmatic Efforts to Promote Children' Safety}

Recommendations, found in the literature, to increase the impact of collective efforts to promote children's safety include, identifying prevalent disparities in Latinx children's representation in the CWS; incorporating Latinx population's diversity within research and program planning; taking in consideration Latinx cultural and socio-economic values into these efforts; and, utilizing approaches that combine social epidemiology with community-engaged research and interventions.

Collective efforts to increase children' safety and to prevent child maltreatment and neglect that partner with and integrate Latinx immigrant families in planning and program delivery efforts must make intentional efforts to identify prevalent disparities in the Child Welfare System (CWS), with the purpose of effectively addressing disproportionality where it exits. It is also important to address the limited availability of data on Latinx families that is disaggregated by various characteristics (e.g., country of origin, generation, socio-economic status, acculturation level, etc.) as opposed to treat Latinx as a monolithic group [30]. Equally important is to ensure that actions to promote children' safety within Latinx communities take in consideration both attitudinal and behavioral components of cultural and socio-economic values [37]. Without this careful and systematic consideration of cultural values, our programmatic efforts to affect Latinx communities may continue to lag behind those efforts to affect non-Latinx Whites in terms of feasibility, acceptability, and effectiveness [37].

Recommendations to partner with and to integrate Latinx families in efforts to promote children' safety made ground for a potential synergy between social epidemiology and community-engaged interventions. Social epidemiology focuses on etiology and distribution of social determinants, and community-engaged interventionists emphasize community rather than population and assume that people live in complex, interconnected, and dynamic contexts [3]. Practitioners and researchers, from these two disciplines, could collaborate with Latinx communities in efforts to characterize disproportionalities in Latinx children's participation in CWS, by building on Latinx diversity and the socio-ecological contexts that influence Latinx children's development and personal safety. As stated by Kimbrough Melton RJ, et al. [18], "the universal hopes and values embedded in the Golden Rule may provide the motivation for concerted action, in diverse contexts, to keep kids safe through new and culturally relevant norms of mutual assistance and individual and collective responsibility to help parents in care for their own and their neighbors' children".

\section{Conclusion}

The United States population is increasingly diverse, particularly with respect to the rapidly growing number of multicultural, multi-lingual, low-income children and families [25]. Cultural diversity and pluralism have significant implications for services delivery and policymaking [24] in child maltreatment and neglect prevention; and makes necessary the study of families' cultural and socioeconomic values to identify aspects of parenting and social networking that are unique to immigrant communities. Strong communities as a multi-level, assets-based and socioecological approach to preventing child maltreatment and neglect, that incorporates community engagement and capacity building strategies, could facilitate development of culturally and linguistically relevant interventions to promote meaningful engagement and to reduce Latinx immigrant families' historic mistrust in outside institutions.

In addition to the historical mistrust sometimes expressed by the Latinx population in the U.S [13], this population accounts for higher poverty rates that nonLatinx Whites [28]. Facing these challenges requires the use of approaches that build on the collectivistic nature and cultural values of Latinos, such as familism. Strategies to promote Latinx community engagement and promoting bonding social capital might contribute to reducing children's risk of maltreatment and neglect. Strong Communities include parents' involvement in their communities, formal and informal social support and interventions to increase social cohesion and mutual support among neighbors. In addition, Strong Communities has been found to be an effective initiative in low-resource communities [5]. Capacity building programs and policies that pursue Latinx families' child maltreatment and neglect prevention should be holistic, multilevel and community-engaged approaches that strengthen children's family environment to enhance children' safety [7]. Such approaches should also incorporate Latinx families and communities' cultural values and assets, 


\section{Epidemiology International Journal}

what makes Strong Communities an applicable initiative to improve children's outcomes in Latinx immigrant communities.

\section{Acknowledgements}

The author developed the first version of this paper in the context of a blue-ribbon international group of scholars' (Kempe-Haruv Fellows) meeting to re-design the United States' child protection policy. Dr. Gary B. Melton coordinated and led this meeting. The meeting was held in South Carolina, in March 2020, organized by The Kempe Foundation with The Kempe Center and The Haruv Institute. I would like to acknowledge the contributions and thoughts provided by Dr. Gary Melton, and other participant scholars in the conceptualization of this paper.

\section{References}

1. El Sayed A (2020) Communities enduring racism \& poverty will suffer most due to COVID-19. Democracy Now.

2. Laster Pirtle WN (2020) Racial Capitalism: A Fundamental Cause of Novel Coronavirus (COVID-19) Pandemic Inequities in the United States. Health Educ Behav 47(4): 504-508.

3. Wallerstein NB, Yen IH, Syme SL (2011) Integration of Social Epidemiology and Community-Engaged Interventions to Improve Health Equity. Am J Public Health 101(5): 822-830.

4. McDonell JR, Ben Arieh A, Melton GB (2015) Strong Communities for Children: Results of a multi-year community-based initiative to protect children for harm. Child Abuse Negl 41: 79-96.

5. McLeigh J, Melton GB (2015) Introduction: Children safety in community context. Child Abuse Negl 41:1-2.

6. (2015) Hispanic Heritage Month 2015. Profile America Facts for Features: CB15-FF.18 (September 15, 2015 Release). United States Census Bureau.

7. Melton GB (2018) Children's Right to Personal Security: the Foundation for a New Journal. Int J Child Maltreat: Research Policy and Practice 1: 1-10.

8. Krogstad J, Lopez M (2015) Hispanic population reaches record 55 million, but growth has cooled. Pew Research Center.

9. Turner K, Wildsmith E, Guzman L, Hammond MA (2016) The Changing Geography of Hispanic Children and Families. The National Research Center on Hispanic
Children and Families.

10. Beckles G, Chou CF (2013) Diabetes-United States, 2006 and 2010. MMWR Suppl 62(03): 99-104.

11. Graham G (2014) Population-based approaches to understanding disparities in cardiovascular disease risk in the United States. Int J Gen Med 7: 393-400.

12. Jack KM, Klein S (2015) Parenting and proximity to social services: Lessons from Los Angeles County in the community context of child neglect. Child Abuse Negl 45: $35-45$.

13. Martinez I, Pokras OC, Brown PB (2009) Addressing the Challenges of Latino Health Research: Participatory Approaches in an Emergent Urban Community. J Natl Med Assoc 101(9): 908-914.

14. Alkire S (2003) A conceptual Framework for Human Security. CRISE Working paper.

15. (1994) Human Development Report. New York: Oxford University Press. United Nations Development Program.

16. (2008) Closing the gap in a generation: health equity through action on the social determinants of health. Commission on Social Determinants of Health [CSDH].

17. Gasper D (2005) Securing humanity: Situating 'Human Security' as concept and discourse. Hum Dev 6(2): 221245.

18. Kimbrough Melton RJ, Melton GB (2015) Someone will notice, and someone will care": How to build Strong Communities for Children. Child Abuse Negl 41: 67-78.

19. Yang MY (2015) The effect of material hardship on child protective service involvement. Child Abuse Negl 41: 113-125.

20. McLeigh JD, McDonell JR, Lavenda O (2018) Neighborhood poverty and child abuse and neglect: The mediating role of social cohesion. Child Youth Serv Rev 93: $154-160$.

21. Jack KM, Lanier P, Motoyama MJ, Welch H, Dineen M (2015) Geographic variation in racial disparities in child maltreatment: The influence of county poverty and population density. Child Abuse Negl 47: 1-13.

22. Koh H, Graham G, Glied S (2011) Reducing racial and ethnic disparities: The action plan from the Department of Health and Human Services. Health Aff 30(10): 18221829.

23. (2020) U.S. Department of Health \& Human Services, 
Administration for Children and Families, Administration on Children, Youth and Families, Children's Bureau (2020) Child Maltreatment 2018.

24. Spector RE (2009) Cultural diversity in health and illness. $7^{\text {th }}$ (Edn.), Pearson Education, Inc, Upper Saddle River, NJ.

25. Lopez M, Hofer KG, Bumgarner E, Taylor D (2017) Developing culturally responsive approaches to serving diverse populations: a resource guide for communitybased organizations. The National Research Center on Hispanic Children \& Families.

26. Kim H, Drake B (2018) Child maltreatment risk as a function of poverty and race/ethnicity in the USA. Int J Epidemiol 47(3): 780-787.

27. Pelton LH (2015) The continuing role of material factors in child maltreatment and placement. Child Abuse Negl 41: 30-39.

28. Edwards A (2019) Hispanic poverty rate hit an all-time low in 2017. United Sates Census Bureau.

29. Tyrone C, Celia CC (2013) Racial disparity in risk factors for substantiation of child maltreatment. Child Youth Serv Rev 35(12): 1962-1969.

30. Motoyamaa MJ, Dettlaffb AJ, Finnoc M (2012) Parental nativity and the decision to substantiate: Findings from a study of Latino children in the second National Survey of Child and Adolescent Well-being (NSCAW II). Child Youth Serv Rev 34(11): 2229-2239.

31. McLeigh JD, McDonell JR, Melton GB (2015) Community differences in the implementation of Strong Communities for Children. Child Abuse Negl 41: 97-112.

32. Scarinci IC, Beech BM, Kovach KW, Bailey TL (2003) An examination of sociocultural factors associated with cervical cancer screening among low-income Latina immigrants of reproductive age. J Immigr Health 5(3): 119-128.

33. Hayden J (2009) Introduction to health behavior theory. Sudbury, Jones and Bartlett Publishers, MA.

34. Zea MC, Quezada T, Belgrave FZ (1994) Latino culture values: Their role in adjustment to disability. Journal of Soc Behav Pers 9(5): 185-200.

35. Baumrind D (1995) Childrearing dimensions relevant to child maltreatment: Child maltreatment and optimal caregiving in social contexts. Garland, New York, pp: 5573.

\section{Epidemiology International Journal}

36. Johnson CE, Mues KE, Mayne SL, Kiblawi AN (2008) Cervical cancer screening among immigrants and ethnic minorities: a systematic review using the health belief model. J Low Genit Tract Dis 12(3): 232-241.

37. Calzada EJ (2010) Bringing culture into parent training with Latinos. Cogn Behav Pract 17(2): 167-175.

38. Bowlby J (1982) Attachment and loss: $2^{\text {nd }}$ (Edn.), New York: Basic Books, Vol 1.

39. Mikulincer M, Shaver PR (2007) Boosting attachment security to promote mental health, prosocial values, and inter-group tolerance. Psychol Inq 18(3): 139-156.

40. Zayas LH, Solar, F (1994) Early childhood socialization in Hispanic families: Context, culture, and practice implications. Prof Psychol Res Pr 25(3): 200-206.

41. Siatkowski A (2007) Hispanic acculturation: a concept analysis. J Transcult Nurs 18(4): 316-323.

42. Marín G, Marín BVO (1991) Research with Hispanic populations. Sage, Applied social research methods series. California.

43. Sabogal F, Marín G, Sabogal RO, Marín BV, Perez Stable EJ (1987) Hispanic familism and acculturation: what changes and what doesn't?. Hisp J Behav Sci 9(4): 397412.

44. Gaines S, Marelich W, Bledsoe K, Steers WN, Henderson MC, et al. (1997) Links between race/ethnicity and cultural values as mediated by racial/ethnic identity and moderated by gender. J Pers Soc Psychol 72(6): 14601476.

45. Michael YL, Farquhar SA, Wiggins N, Green MK (2008) Findings from a Community-based Participatory Prevention Research Intervention Designed to Increase Social Capital in Latino and African American Communities. J Immigr Minor Health 10(3): 281-289.

46. Moore de Peralta A, Davis L, Brown K, Fuentes M, Falconer NS, et al. (2019) Using community-engaged research to explore social determinants of health in a low-resource community in the Dominican Republic: A community health assessment. Hisp Health Care Int 18(3): 127-137.

47. Sandoval JA, Lucero J, Oetzel J, Avila M, Belone L, et al. (2012) Process and outcome constructs for evaluating community-based participatory research projects: a matrix of existing measures. Health Educ Res 27(4): 680-690.

48. Ahmed SM, Palermo AG (2010) Community engagement in research: frameworks for education and peer review. 


\section{Epidemiology International Journal}

Am J Public Health 100(8): 1380-1387.

49. Lucero JE, Wallerstein N (2013) Trust in communityacademic research partnerships: Increasing the consciousness of conflict and trust development. In:
Oetzel JG, Toomey ST (Editors.), The Sage Handbook of Conflict Communication: Integrating Theory, Research, and Practice, Sage Publications Thousand Oaks, CA, USA, pp: 537-561. 